\title{
Comparison of major minerals uptake between conventional and non-tillage/non-fertilizer/non-chemical vegetables in Japan
}

\author{
M. Funabashi \\ Sony Computer Science Laboratories, Inc., Tokyo, Japan
}

According to the nutrition standard database in Japan, trophic factors of vegetables such as vitamin, iron, minerals are known to decrease after 1950 with the rise of conventional agriculture ${ }^{(1)}$. This change is correlated with the increased use of chemical fertilizer and practice of intensive monoculture as conventional method ${ }^{(2)}$. This study tries to compare the effect of ecologically different cultivation methods on minerals concentration in vegetables, between conventional and non-tillage/non-fertilizer/non-chemical farming.

We have established a new cultivation system, namely Synecoculture, based on a dense mixture of plural vegetables in open culture without tillage/fertilizer/chemical to produce a closely natural state metabolism of vegetable and conformation of soil ${ }^{(3)}$. We analyzed 140 samples from 37 vegetable species and measured the quantity of 4 major ions: $\mathrm{Na}, \mathrm{K}, \mathrm{Ca}, \mathrm{Mg}$, both of vegetables and field soil with atomic absorption spectrophotometer.

The results of soil ion analysis showed the field concentration is qualitatively in the same level as surrounding natural grassland, meaning a part of minerals are not sufficient/unbalanced compared to the standard of conventional method. The soil was Mg defficient with respect to the standard protocol of Japanese Agricultural Cooperatives (JA), which was also confirmed by an independent analysis by a local JA branch (Fig. 1). Nevertheless, the concentration of $\mathrm{Na}, \mathrm{Ca}, \mathrm{Mg}$ in vegetables showed higher proportional average than Japanese standards (Fig. 2).

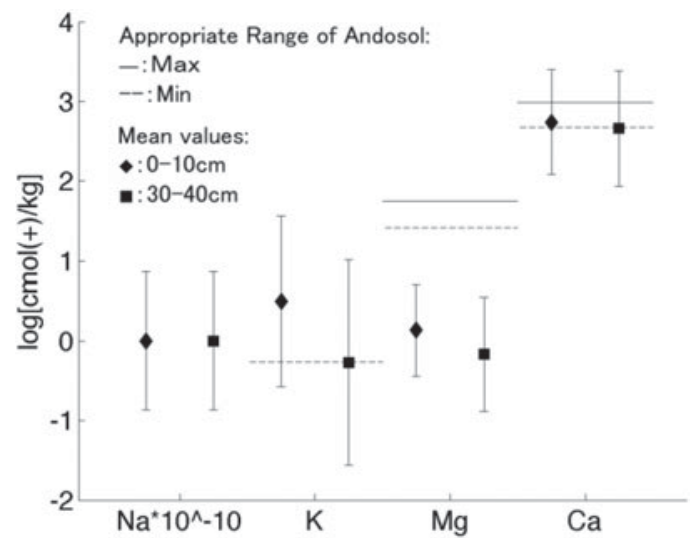

Fig. 1. Soil minerals analysis.

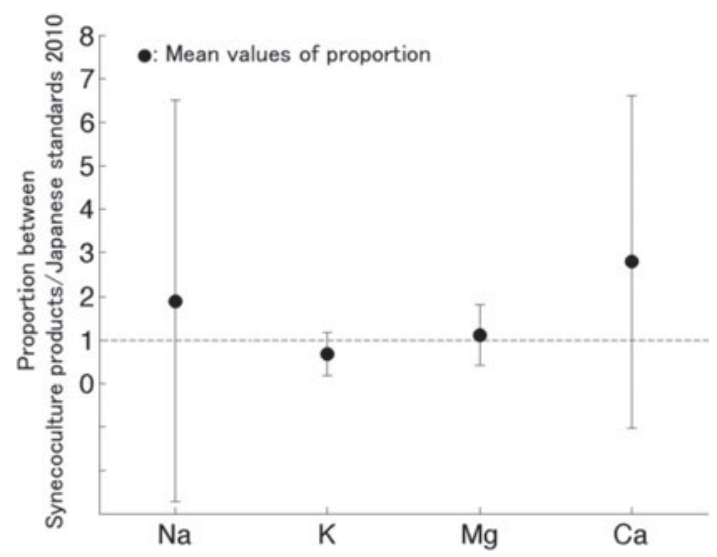

Fig. 2. Vegetable minerals analysis.

Although the soil minerals are not sufficient/unbalanced with respect to the conventional standard, vegetables raised with dense polyculture under non-tillage/non-fertilizer/non-chemical condition tend to accumulate even superior content of minerals in a long term average. This results explain the optimization of conventional method is based on the quantity of harvest and not on the food components such as minerals, which is considered to play important role in long-term human health.

1. Ministry of Education, Culture, Sports, and Technology-Japan (1950, 1963, 1980, 2005, 2010) Standard Tables of Food Composition in Japan.

2. Michinori N (2002) Journal of the Science of Soil and Manure, Japan 73(2), 219-225.

3. Funabashi M (2011) Actes du colloque "Transversalités de l'Agriculture Biologique". 